\title{
La diferenciación espacial del envejecimiento demográfico de la Comunidad Autónoma de Madrid
}

\author{
M. ${ }^{a}$ José Aguilera Arilla y M. ${ }^{a}$ Pilar González Yanci
}

\section{INTRODUCCION}

En 1986 se celebró en Estrasburgo un Seminario sobre las Regiones con problemas demográficos en Europa. Una de sus conferencias versó sobre el envejecimiento como uno de los más destacables. Esto no es más que un ejemplo de la preocupación que existe por este fenómeno, que es nuevo en la Historia de la Humanidad y que aparece vinculado a los paises que han alcanzado un cierto nivel de desarrollo. Las consecuencias que se pueden derivar de este hecho han motivado un creciente interés por estudiarlo desde diversos enfoques, que tratan en unos casos de definir el fenómeno, en otros de estudiar sus causas y consecuencias, de predecir situaciones futuras, o de analizar su distribución espacial.

El analizar la distribución espacial es sin duda uno de los primeros pasos a dar en la explicación global del fenómeno. Evidentemente la homogeneidad no existe en ninguna de las características demográficas de la población. Es cierto que se observa una tendencia al envejecimiento en todos los países desarrollados, pero existen notables diferencias entre unos y otros y aún dentro de cada país pueden observarse contrastes entre unas regiones y otras y entre los medios rural y urbano.

El estudio del profesor Paillat presentado en el Seminario citado anteriormente da una idea de la situación de los países de Europa Occidental. En el cuadro I puede verse que en torno a 1980 la mayoría de los países citados superan o están muy próximos al 12 por 100 de ancianos en el total de la población, siendo incluso numerosos los que están por encima de la 
cifra del 14 por 100 . Sólo Islandia e Irlanda se mantienen a cierta distancia de la barrera del 12 por 100 considerada como límite para el envejecimiento por varios autores.

Comparando con los datos de 1970 puede verse cómo además el proceso avanza a un ritmo rápido, pues en aquella fecha tan sólo un país, Austria, había superado la barrera del 14 por 100 , mientras que cuatro países estaban aún por debajo del 10 por 100. Observando en el mismo cuadro la

CUADRO I. EVOLUCION DEL \% DE ANCIANOS Y RELACION V/J EN EUROPA OCCIDENTAL

\begin{tabular}{|c|c|c|c|c|c|c|}
\hline PAIS & AÑO & $\% \vee$ & $\mathrm{V} / \mathrm{J}$ & AÑO & $\% \vee$ & $V / J$ \\
\hline \multicolumn{7}{|l|}{ P. ESCANDINAVOS } \\
\hline Islandia . . . . . . . . & 1970 & 8,9 & 27,6 & 1980 & 9,5 & 34,9 \\
\hline Noruega .... & $"$ & 11,2 & 43,4 & $"$ & 12,9 & 55,1 \\
\hline Suecia ........... & $"$ & 13,6 & 65,1 & $"$ & 16,6 & 86,9 \\
\hline Finlandia & 1972 & 9,9 & 42,9 & 1982 & 12,3 & 62,4 \\
\hline Dinamarca ... & 1975 & 13,4 & 59,0 & 1985 & 15,0 & 80,6 \\
\hline \multicolumn{7}{|l|}{ ISLAS BRITANICAS } \\
\hline Irlanda ....... & 1971 & 11,1 & 35,5 & 1980 & 10,7 & 35,3 \\
\hline Escocia ....... & " & 12,0 & 45,3 & $"$ & 14,1 & 65,9 \\
\hline Inglaterra & 1978 & 13,7 & 58,1 & $"$ & 15,2 & 74,9 \\
\hline Gales ............ & 1970 & 13,6 & 57,6 & $"$ & 15,3 & 75,4 \\
\hline Ulster . ........... & " & 11,0 & - & $"$ & 11,9 & 44,7 \\
\hline \multicolumn{7}{|l|}{ BENELUX } \\
\hline Paises Bajos & 1974 & 10,6 & 40,6 & 1984 & 11,9 & 58,3 \\
\hline Bélgica .......... & 1970 & 13,4 & 56,8 & 1981 & 14,4 & 72,0 \\
\hline Luxemburgo ....... & & - & - & & - & - \\
\hline Rep. Fed. Alemania & 1970 & 13,2 & 56,8 & 1983 & 14,6 & 91,8 \\
\hline Francia .......... & 1968 & 13,4 & 56,5 & 1982 & 13,8 & 66,7 \\
\hline Portugal & 1970 & 9,7 & 34,2 & 1981 & 11,4 & 44,7 \\
\hline España ........... & 1970 & 9,7 & 34,9 & 1981 & 11,3 & 44,1 \\
\hline Italia $\ldots \ldots \ldots \ldots$ & 1971 & 11,3 & 46,1 & 1981 & 13,7 & 64,6 \\
\hline Suiza $\ldots \ldots \ldots \ldots$ & 1970 & 11,4 & 48,7 & 1980 & 13,9 & 66,1 \\
\hline Austria $\ldots \ldots \ldots$ & 1971 & 14,2 & 58,6 & 1981 & 15,2 & 76,0 \\
\hline Grecia & $"$ & 11,0 & 43,3 & 1981 & 12,8 & 54,0 \\
\hline
\end{tabular}

Fuente: PAILLAT (1987). 
relación entre viejos y jóvenes ${ }^{1}$ se pone de manifiesto que la situación real está agravada por el hecho de que los jóvenes son cada vez menos numerosos, dado que hay un descenso general de la natalidad.

En el conjunto europeo España aparece como uno de los países menos envejecidos, en 1970 tan sólo tenía un 9,7 por 100 de mayores de 65 años. En 1981 el porcentaje se habia incrementado hasta un 11,3 por 100. El retraso en el proceso de industrialización es la causa más clara del desfase con respecto al resto de los países europeos. Pero aún así es evidente que el proceso de envejecimiento está en marcha. De nuevo se pueden constatar notables contrastes entre Comunidades autónomas: hay una coincidencia entre Comunidades costeras y bajo grado de envejecimiento y Comunidades interiores y alto grado de envejecimiento. Tomando como referencia la media nacional, la sobrepasan ampliamente Aragón, Galicia, Castilla-La Mancha y La Rioja, mientras que están por debajo Canarias, País Vasco, Madrid, Murcia y Andalucía. Dos excepciones aparecen claras, el caso de Galicia, que siendo Comunidad costera está entre las de alto grado de envejecimiento y la de Madrid, que, situada en el interior tiene un nivel bajo.

El objetivo de este estudio es analizar, precisamente, el caso de la Ccmunidad de Madrid, que ofrece un interés especial por incluir la más importante ciudad del Estado.

En un trabajo aún inédito estudiamos el envejecimiento por barrios de la capital. En él se constató la existencia de un proceso de envejecimiento especialmente acusado en los barrios centrales. Pudimos comprobar que, en efecto, la distancia al centro se traducía en un menor porcentaje de ancianos, lo que representado de forma matemática se ajusta a una curva de regresión.

Hay por tanto dentro de una ciudad una diferenciación espacial nítida que obedece a condicionamientos muy variados de carácter económico, social, cultural, etc. Si ampliamos el área de estudio a toda la Comunidad madrileña obtendremos, sin duda, nuevos contrastes espaciales. Analizar estos contrastes es nuestro presente objetivo.

Téngase en cuenta que en el cuadro el resultado de la relación $\mathrm{V} / \mathrm{J}$ está multiplicada por cien, mientras que en el mapa de la provincia de Madrid no lo está. 


\section{EL ENVEJECIMIENTO POR MUNICIPIOS EN 1981}

Utilizando los datos que nos brinda el Censo de 1981 hemos elaborado una serie de mapas para ver la distribución espacial del envejecimiento en la Comunidad Autónoma tomando como base el término municipal.

La representación del porcentaje de ancianos (mapa 1) muestra el contraste entre un área central poco o nada envejecida, frente a unos extremos envejecidos aunque con diferencias entre ellos. El área central, que abarca un elevado número de municipios, es extensa en relación al pequeño tamaño de la provincia (la Comunidad de Madrid es uniprovincial). Incluye la capital y su área metropolitana, y ésta es precisamente la razón de la "juventud» de la zona. En las grandes ciudades europeas como Londres o París se repite esta situación, a pesar de los elevados indices de envejecimiento nacionales, las capitales aparecen rodeadas de circunscripciones más jóvenes, que constituyen su área metropolitana y que «rejuvenecen» el conjunto. (PAILLAT, 1987).

En torno al área central hay tres zonas con porcentajes superiores al 12 por 100 de ancianos. Las situadas al Oeste y SE., respectivamente presentan una situación intermedia, no superando, salvo la excepción de Ambite, el 24 por 100. La zona Norte, sin embargo, presenta mayor variedad, con valores muy elevados de envejecimiento; hay municipios que superan el 36 por 100 de ancianos e incluso en el caso de la Hiruela el 50 por 100.

Dentro del área central el municipio de Madrid tiene un valor de 11,2 por 100, uno de los más elevados del conjunto, mientras que su área metropolitana, constituye una aureola en la que los valores son muy bajos, y que ni siquiera supera el 6 por 100. Hay por tanto una situación diferente a la que encontramos en el estudio arriba citado del envejecimiento de los barrios de Madrid. Allí el centro era lo más envejecido y según nos alejábamos de él los valores disminuían. Ahora sucede lo contrario, el área central es más joven que la periferia, pero hay que tener en cuenta un hecho importante: la correlación distancia-juventud se continúa hasta el límite del área metropolitana (considerando no el área metropolitana propiamente dicha, sino la funcional, que incluye, además otros municipios). A partir de aquel límite se produce una inflexión y el envejecimiento va en aumento según nos alejamos del centro. 
Los restantes mapas (números 2 y 3 ) corroboran lo que se ha puesto de manifiesto en el primero. El número 2 se ha confeccionado tomando como referencia la media provincial $(9,3$ por 100$)$ para ver los que están por encima o debajo de ese valor. El número 3 , muy similar al 1, está realizado con el índice de envejecimiento consistente en dividir los mayores de 65 años por los menores de 16 , con lo que se obtiene una importante matización, la de establecer la relación con el número de jóvenes, ya que los municipios viejos tienen pequeña proporción de jóvenes y en cambio los menos envejecidos tienen muchos.

La situación comentada está en consonancia con la de los paises desarrollados occidentales, aunque los valores son claramente inferiores, equivalentes a los que aquéllos tenían en torno a 1970. Es previsible que dentro de unos años la situación española sea similar a la actual de Europa o incluso que lleguen a encontrarse. La evolución experimentada en los últimos años en la Comunidad objeto de nuestro estudio corrobora esta idea.

\section{EVOLUCION DEL PROCESO DE ENVEJECIMIENTO DESDE 1950}

Para ver la evolución del envejecimiento de la Comunidad hemos utilizado los datos publicados en los censos de 1950, 60, 70 y 1981. En los tres primeros la información no se ha publicado a nivel municipal, por lo que hemos utilizado una aproximación por áreas: área rural, área intermedia y área urbana, que están definidas en función del tamaño de su población.

Representando en cada área el porcentaje de ancianos en cada uno de los cuatro censos se han obtenido unos mapas (números $4,5,6$ y 7) que muestran de forma gráfica la evolución que se ha producido. Téngase en cuenta que los municipios comprendidos en cada área no son siempre los mismos sino que algunos han pasado a formar parte de otra área al aumentar su población.

En 1950 la proporción de mayores de 65 años era baja en toda la provincia, con diferencias pequeñas entre las distintas áreas. En 1960 el porcentaje de envejecimiento ha aumentado en conjunto y se mantienen las diferencias entre las áreas algo incrementadas. Entre el área urbana y el área rural hay más de un 1 por 100 de distancia. En 1970 se continúa envejeciendo el conjunto y van aumentando las diferencias entre las áreas. 
Mientras que en el 50 entre la zona urbana y la rural sólo había 0,6 por 100 , en el 70 hay 2,1 por 100. El salto más acusado se produce entre 1970 y 1981 , ahora las diferencias se hacen muy grandes tanto entre los dos censos como entre las tres áreas. La zona rural alcanza un valor elevado (16,1 por 100) que supone un 10 por 100 más con respecto a 1950. En cambio, es de destacar que en la zona urbana se ha producido un gran rejuvenecimiento, pues tras haber tenido valores superiores en 1960 y 1970 vuelve a una situación similar a la de 1950. Hecho que se justifica tan sólo por la inclusión en la zona urbana de los municipios del área metropolitana, pues la capital tiene un valor mucho más elevado (11,2 por 100). Esta situación es idéntica a la de las grandes ciudades europeas comentada anteriormente.

Obsérvese viendo los mapas 4, 5, 6 y 7 y el cuadro II, cómo los porcentajes del área rural en un censo, en el siguiente pasan a la zona intermedia y urbana, hasta que en 1981 se rompe la secuencia, ya que la zona urbana vuelve a los valores que tenía al comienzo.

En el gráfico 1 puede verse con claridad lo que acabamos de comentar, que hay un proceso general de envejecimiento y que desde 1970 se dispara la zona rural, alejándose del resto, mientras que en la zona urbana hay un rejuvenecimiento.

\section{CUADRO II. EVOLUCIÓN DEL PORCENTAJE DE ANCIANOS POR ZONAS EN LA COMUNIDAD DE MADRID}

\begin{tabular}{|c|c|c|c|c|c|c|c|c|}
\hline & \multicolumn{2}{|c|}{1950} & \multicolumn{2}{|c|}{1960} & \multicolumn{2}{|c|}{1970} & \multicolumn{2}{|c|}{1981} \\
\hline & $\mathrm{POB}+65$ & $\%$ & $\mathrm{POB}+65$ & $\%$ & $\mathrm{POB}+65$ & $\%$ & $\mathrm{POB}+65$ & $\%$ \\
\hline TOTAL PROVIN. .. & 114.948 & 5,9 & 181.273 & 7,0 & 296.219 & 7,9 & 439.610 & 9,3 \\
\hline ZONA URBANA & 96.740 & 5,9 & 159.005 & 6,9 & 273.483 & 7,8 & 247.739 & 5,5 \\
\hline ZONA INTERMEDIA & 9.169 & 5,5 & 11.801 & 6,7 & 12.637 & 7,8 & 14.686 & 10,4 \\
\hline ZONA RURAL & 8.339 & 6,5 & 10.467 & 8,1 & 10.114 & 10,0 & 13.218 & 16,1 \\
\hline
\end{tabular}

Fuente: Censo de la Población de España. INE. 


\section{ANALISIS DE LA DIFERENCIACION ESPACIAL DEL ENVEJECIMIENTO}

Vistas las caracteristicas de la distribución espacial y la evolución del envejecimiento vamos a pasar a estudiar con qué aspectos aparece aquél relacionado. Las causas del envejecimiento son claras: el descenso de la fecundidad acompañado de una débil mortalidad lleva a un aumento del número de adultos y ancianos en el conjunto de la población, que unido al crecimiento de la esperanza de vida da lugar a un envejecimiento. Los movimientos migratorios introducen modificaciones importantes. Así, si consideramos la población española en conjunto podemos ver que, en efecto, al descender la fecundidad y ser baja la mortalidad, con el consiguiente aumento de la esperanza de vida, tiene lugar un proceso de envejecimiento, tal como ocurre en todos los países desarrollados en mayor o menor grado. Ahora bien, si descendemos a un nivel de detalle, analizando los valores reales de envejecimiento en circunscripciones menores (provincia, municipio) se ponen de manifiesto acusados contrastes espaciales, como decíamos anteriormente.

En el caso que estudiamos se ha visto ya la existencia de contrastes que están motivados fundamentalmente por un hecho clave: los movimientos migratorios. Éstos, a su vez, son efecto de una multitud de condicionamientos de carácter sociocultural y, sobre todo, económico.

No es objeto de este trabajo el estudio de los movimientos migratorios que han afectado a los municipios, pero para poder ver la relación hemos acudido a la aproximación que supone ver el saldo de la población de cada municipio entre 1960 y 1981, obteniendo un mapa de pérdidas y ganancias de población (mapa 8). El resultado ha sido que hay una coincidencia de los municipios que ganan población con los que se mantienen"más jóvenes 0 se rejuvenecen y de los que pierden con los que se envejecen. Parece claro que la explicación de la distribución espacial del envejecimiento de la Comunidad de Madrid está sobre todo en los movimientos migratorios.

Ahora bien, como hemos dicho, los movimientos migratorios son el resultado de la acción combinada de una serie de variables muy complejas, que van desde la valoración paisajístico-turística hasta las más netamente económicas (encontrar un puesto de trabajo, por ejemplo). Ello nos ha inducido a proceder al estudio de algunas de las variables que en una primera observación parecen poder influir en el proceso. 
No todas son cuantificables ni es posible contemplar la totalidad; en una primera aproximación, que pretendemos continuar en posteriores investigaciones, hemos optado por aquellas para las que teníamos datos en el presente. Estas variables son: el tamaño de la población, la distancia a la capital, el incremento de población entre 1960 y 1981 y el porcentaje de población activa dedicada a la agricultura y a la industria.

Ya hemos visto la relación de la distribución espacial del envejecimiento con el incremento de población entre 1960 y 1981 (mapa 8). Si consideráramos en vez de este largo período el quinquenio 75-81 veríamos que la situación es idéntica, excepto en el caso del municipio de Madrid que empezó a perder población a favor de su área metropolitana. Hecho que destacamos para explicar el mayor índice de envejecimiento que ya hemos comentado en Madrid capital.

Comparando con el porcentaje de población activa dedicada a la agricultura (mapa 9) se aprecia igualmente una correspondencia clara entre los municipios con elevado porcentaje de aquélla y los más envejecidos, con alguna excepción como Villaconejos, que ha conservado una agricultura tradicional de elevados rendimientos (el medio rural expulsa población).

Algo similar ocurre en sentido contrario con los municipios en que hay elevada proporción de activos dedicados a la industria, que coinciden con los menos envejecidos (mapa 10) (la industria atrae a la población).

Los municipios que siendo poco envejecidos tienen relativamente poca actividad en la industria son los que poseen un alto porcentaje de activos dedicados a servicios, como es el caso de los de la zona W-SW del área metropolitana y los de la Sierra de Guadarrama, ciudades dormitorio los primeros y de residencia secundaria los segundos.

Íntimamente relacionado con los movimientos migratorios está el tamaño de la población. El gran foco de atracción es Madrid. A su alrededor ha ido creciendo una nube de poblaciones que albergan a los más jóvenes y muestran crecimientos espectaculares. Estas poblaciones se sitúan fundamentalmente junto a las principales vias de comunicación, que juegan un papel trascendental en la localización de la población. En este caso se da también coincidencia entre las poblaciones de mayor tamaño, que han recibido inmigrantes con las menos envejecidas y las más pequeñas, que han perdido población con las más envejecidas. La coincidencia se da justamente en las poblaciones que han crecido de forma espectacular en los últimos decenios y no con las que ya tenían elevada población desde anti- 
guo, lo que corrobora que son los inmigrantes, en su gran mayoría jóvenes, los que producen el efecto citado.

Una vez visto de forma cartográfica que hay ciertas relaciones, vamos a proceder a analizarlo estadísticamente para comprobar su validez, utilizando los resultados que se obtengan como punto de partida de una auténtica explicación posterior.

En el cuadro III se recoge la información utilizada para los 178 municipios madrileños.

\section{CUADRO III}

\begin{tabular}{|c|c|c|c|c|c|c|}
\hline MUNICIPIO & $\begin{array}{l}\text { POBLACION } \\
\text { TOTAL }\end{array}$ & $\begin{array}{c}\text { DISTANCIA } \\
\text { MADRID }\end{array}$ & $\begin{array}{c}\% \\
\text { INCREMENTO } \\
1960-81\end{array}$ & AGRICULTURA & $\stackrel{\%}{\%}$ & $\begin{array}{c}\% \\
\text { ANCIANOS }\end{array}$ \\
\hline Acebeda & 70 & 90 & $-65,2$ & 74,5 & 0 & 36,9 \\
\hline Ajalvir & 972 & 25 & 6,8 & 29,8 & 36,9 & 9,7 \\
\hline Alameda $V . \ldots \ldots \ldots$ & 150 & 92 & -55 & 76 & 0 & 26,4 \\
\hline Alamo ..... & 2.407 & 37 & 101,9 & 15 & 14,1 & 10,6 \\
\hline Alcalá de Henares .... & . 142.862 & 30 & 468,7 & 1,9 & 44,3 & 5 \\
\hline Alcobendas $\ldots \ldots \ldots \ldots$ & 63.507 & 17 & $1.229,1$ & 2,2 & 35,8 & 3,9 \\
\hline Alcorcón ... & 140.657 & 13 & $4.091,2$ & 0,8 & 28 & 4,2 \\
\hline Aldea del Fresno & 85 & 45 & 23,8 & 21,7 & 9,8 & 9,8 \\
\hline Algete $\ldots \ldots \ldots$ & 5.042 & 30 & 338,4 & 14 & 37,3 & 5 \\
\hline Alpedrete & 2.392 & 40 & 52,8 & 8,8 & 21,3 & 8,7 \\
\hline Ambite.. & 613 & 53 & $-18,4$ & 34,9 & 5 & 27,3 \\
\hline Anchuelo & 518 & 37 & $-7,3$ & 27 & 37,9 & 11,1 \\
\hline Aranjuez & 35.936 & 47 & 31,9 & 9,7 & 41,2 & 10,7 \\
\hline Arganda & 21.940 & 27 & 249,5 & 7,4 & 42,7 & 7,6 \\
\hline Arroyomolinos & 209 & 22 & $-2,3$ & 77,8 & 0 & 9,1 \\
\hline Atazar ...... & 74 & 65 & $-35,1$ & 40 & 20 & 23 \\
\hline Batres & 181 & 35 & $-15,4$ & 25 & 16,7 & 17,5 \\
\hline Becerril & 1.403 & 50 & 62,2 & 5,1 & 12,7 & 10,2 \\
\hline Belmonte .. & 1.082 & 55 & $-20,1$ & 22,4 & 31,5 & 14 \\
\hline Berrueco... & 201 & 65 & -47 & 46,4 & 22,7 & 21 \\
\hline Berzosa . & 53 & 80 & -46 & 63,5 & 0 & 28,7 \\
\hline Boadilla del Monte ..... & 6.061 & 16 & 446 & 6,7 & 21,9 & 5,3 \\
\hline Boalo ........... & 1.256 & 51 & 34,8 & 32,3 & 14,9 & 8,3 \\
\hline Braojos ... & 136 & 81 & $-51,9$ & 40,2 & 0 & 11,6 \\
\hline Brea del Tajo & 481 & 62 & $-48,3$ & 39,6 & 8,1 & 21,5 \\
\hline Brunete ..... & 1.119 & 30 & 24,8 & 21,4 & 21,5 & 12,1 \\
\hline Buitrago & 1.267 & 76 & 71,9 & 9,7 & 11,1 & 9,2 \\
\hline Bustarviejo & 1.051 & 55 & $-20,9$ & 47,5 & 14,4 & 18,1 \\
\hline Cabanillas & 543 & 54 & 84,1 & 35,7 & 0 & 15,6 \\
\hline Cabrera... & 819 & 60 & 55,1 & 20 & 15,6 & 11,2 \\
\hline Cadalso de los Vidrios & 2.164 & 75 & $-2,4$ & 25,8 & 11,5 & 18,3 \\
\hline Camarma $\ldots \ldots \ldots \ldots$ & 1.288 & 36 & 89,4 & 12,7 & 42,2 & 7,6 \\
\hline
\end{tabular}




\section{CUADRO III (Continuación)}

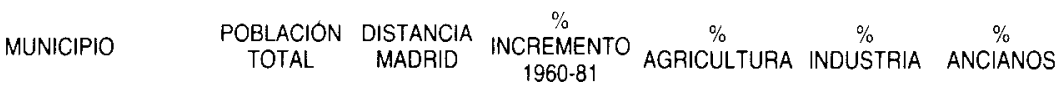

\begin{tabular}{|c|c|c|c|c|c|c|}
\hline Campo Real & 2.025 & 33 & 5,4 & 10,6 & 42,9 & 11,5 \\
\hline Canencia $\ldots \ldots \ldots \ldots$ & 466 & 67 & $-18,6$ & 35,8 & 0 & 20,3 \\
\hline Carabaña $\ldots \ldots \ldots \ldots$ & 1.060 & 50 & $-45,6$ & 23,6 & 21,2 & 20,8 \\
\hline Casarrubuelos & 520 & 31 & 4,6 & 31,4 & 22,9 & 11,4 \\
\hline Cenicientos $\ldots \ldots \ldots \ldots$ & 2.403 & 79 & $-4,1$ & 70,2 & 9,1 & 19,2 \\
\hline Cercedilla ............ & 3.972 & 59 & 11 & 7,7 & 5,1 & 9,8 \\
\hline Cervera B. $\ldots \ldots \ldots \ldots$ & 100 & 60 & $-53,5$ & 14,6 & 0 & 27,2 \\
\hline Ciempozuelos & 10.268 & 32 & 13,6 & 24 & 31,3 & 11,9 \\
\hline Cobeña ....... & 582 & 25 & 70,2 & 21,2 & 42,5 & 8,2 \\
\hline Colmenar de Oreja & 5.004 & 50 & $-9,8$ & 22,1 & 24,8 & 15,1 \\
\hline Colmenar de $\mathrm{A}$. & 416 & 50 & $-24,1$ & 19,3 & 22,6 & 21 \\
\hline Colmenar Viejo & 21.159 & 31 & 152,6 & 8,9 & 22,6 & 9,6 \\
\hline Colmenarejo . . & 1.595 & 35 & 164,1 & 9,7 & 8,3 & 8,2 \\
\hline Collado Villalba & 18.700 & 38 & 244,7 & 2,5 & 32,5 & 5,4 \\
\hline Collado Mediano & 1.547 & 49 & 48,9 & 5,7 & 18,6 & 8 \\
\hline Corpa & 369 & 43 & $-31,2$ & 31,8 & 18,6 & 15,3 \\
\hline Coslada & 53.289 & 12 & $1.342,2$ & 0,8 & 47,8 & 3,1 \\
\hline Cubas & 788 & 28 & 93,1 & 10 & 20 & 19,6 \\
\hline Chapinería & 601 & 50 & $-15,6$ & 24,6 & 19,2 & 15,6 \\
\hline Chinchón & 3.900 & 45 & -12 & 33,6 & 24,7 & 16,7 \\
\hline Daganzo $A \ldots \ldots \ldots$ & 1.042 & 27 & 47,8 & 20,7 & 35,6 & 8 \\
\hline Escorial. & 6.192 & 50 & 64,5 & 9,1 & 15,9 & 10 \\
\hline Estremera ....... & 1.106 & 62 & $-39,5$ & 38,1 & 24,1 & 18,7 \\
\hline Fresnedillas $\ldots \ldots \ldots \ldots$ & 449 & 49 & $-6,5$ & 20 & 10 & 15 \\
\hline Fresno de Torote & 102 & 38 & $-44,3$ & 55,3 & 0 & 6,9 \\
\hline Fuenlabrada..... & 77.626 & 18 & $2.632,4$ & 5,2 & 39,1 & 1,9 \\
\hline Fuente el Saz ... & 1.421 & 32 & 66,8 & 27 & 17,6 & 9,3 \\
\hline Fuentidueña & 1.184 & 62 & -23 & 33,9 & 18,7 & 14,4 \\
\hline Galapagar & 6.090 & 33 & 148,3 & 6,4 & 9,1 & 7 \\
\hline Garganta de los Montes & 310 & 78 & $-38,5$ & 68,3 & 0 & 16,7 \\
\hline Gargantilla de Lozoya & 224 & 80 & $-44,7$ & 62,5 & 0 & 16,3 \\
\hline Gascones & 82 & 80 & $-24,1$ & 33,2 & 0 & 15,5 \\
\hline Getafe .... & 127.060 & 13 & 480,3 & 1,3 & 46,3 & 4,8 \\
\hline Griñón .... & 1.311 & 28 & 21,6 & 12,6 & 47 & 12,1 \\
\hline Guadalix & 1.755 & 45 & 22,3 & 38,7 & 21,5 & 14,7 \\
\hline Guadarrama & 6.682 & 48 & 76,5 & 5,9 & 6,5 & 6,9 \\
\hline Hiruela & 35 & 105 & $-82,3$ & 100 & 0 & 53,1 \\
\hline Horcajo & 148 & 88 & $-63,3$ & 91,5 & 0 & 39,9 \\
\hline Horcajuelo & 60 & 90 & $-64,3$ & 63,5 & 36,5 & 28,3 \\
\hline Hoyo de Manzanares ... & 3.476 & 36 & 81,4 & 13,1 & 0,8 & 7 \\
\hline Humanes $M . \ldots \ldots \ldots$ & 2.985 & 22 & 259,6 & 22,4 & 53,1 & 5,2 \\
\hline Leganés $\ldots \ldots \ldots \ldots$. & 163.426 & 12 & $1.813,9$ & 1,1 & 38,1 & 3,8 \\
\hline Loeches . & 2.004 & 30 & 16,6 & 2,9 & 51,5 & 9,3 \\
\hline Lozoya & 453 & 85 & $-22,7$ & 24,2 & 21,2 & 20,1 \\
\hline Lozoyuela N, S & 605 & 68 & $-29,8$ & 23,5 & 21,5 & 17,3 \\
\hline Madarcos .... & 43 & 85 & $-59,1$ & 49,7 & 0 & 21,6 \\
\hline Madrid ... & 3.188 .297 & 0 & 41,1 & 0,7 & 26,3 & 11,2 \\
\hline Majadahonda & 22.949 & 18 & 654,4 & 3,4 & 13,1 & 3,8 \\
\hline Manzanares. & 1.575 & 48 & 88,2 & 8,2 & 14,1 & 8,2 \\
\hline Meco ..... & 1.144 & 38 & 19,5 & 22,7 & 34,6 & 9,4 \\
\hline
\end{tabular}




\section{CUADRO III (Continuación)}

\begin{tabular}{|c|c|c|c|c|c|c|}
\hline MUNICIPIO & $\begin{array}{l}\text { POBLACION } \\
\text { TOTAL }\end{array}$ & $\begin{array}{l}\text { DISTANCIA } \\
\text { MADRID }\end{array}$ & $\begin{array}{c}\% \\
\text { INCREMENTO } \\
1960-81\end{array}$ & $\stackrel{\%}{\%}$ AGRICULTURA & INDUSTRIA & $\begin{array}{c}\% \\
\text { ANCIANOS }\end{array}$ \\
\hline Mejorada del Campo & 9.519 & 18 & 306,8 & 10,5 & 40,3 & 4 \\
\hline Miraflores de la Sierra & 2.334 & 50 & 21,9 & 14,9 & 11 & 11,4 \\
\hline Molar $(E I) \ldots \ldots \ldots$ & 2.690 & 42 & 45,7 & 17,5 & 18,3 & 11,8 \\
\hline Molinos (Los) & 2.145 & 50 & 26,8 & 9,6 & 8 & 12,8 \\
\hline Montejo de la Sierra & 198 & 82 & $-48,2$ & 50 & 12,5 & 28,4 \\
\hline Moraleja .......... & 1.035 & 23 & 69,7 & 28,3 & 28,3 & 8,3 \\
\hline Moralzarzal & 1.567 & 46 & 86,3 & 6,7 & 5,2 & 8,6 \\
\hline Morata de Tajuña & 4.546 & 35 & 19,6 & 23,2 & 28,6 & 11,8 \\
\hline Móstoles ....... & 149.649 & 18 & $5.085,3$ & 1,8 & 33,1 & 2,8 \\
\hline Navacerrada & 1.270 & 50 & 113,8 & 14,8 & 7,4 & 7,2 \\
\hline Navalafuente & 384 & 58 & 50,6 & 41,7 & 16,7 & 19,4 \\
\hline Navalagamella & 508 & 47 & 1,6 & 43 & 2,7 & 17,9 \\
\hline Navalcarnero . & 8.034 & 31 & 71,6 & 14,5 & 30,8 & 10 \\
\hline Navarredondo & 121 & 85 & $-61,9$ & 25,3 & 17 & 20,2 \\
\hline Navas del Marqués & 867 & 51 & 7 & 13,7 & 17,2 & 16,5 \\
\hline Nuevo Baztán .... & 461 & 47 & 104,9 & 12,5 & 4,2 & 7,5 \\
\hline Olmeda $\mathrm{F}^{\prime} \ldots$ & 152 & 49 & $-54,2$ & 41,7 & 0 & 20,1 \\
\hline Orusco & 579 & 55 & $-45,1$ & 32,6 & 26 & 21,9 \\
\hline Paracuellos del Jarama & 2.828 & 18 & 91 & 12,6 & 24,8 & 6,8 \\
\hline$\ldots \ldots \ldots \ldots \ldots \ldots$ & 55.933 & 20 & $3.040,5$ & 1,8 & 45,7 & 2,5 \\
\hline Patones & 353 & 60 & $-3,3$ & 41,1 & 13,8 & 12,3 \\
\hline Pedrezuela & 727 & 45 & 26 & 8,6 & 20 & 13,9 \\
\hline Pelayos de la Presa & 651 & 63 & 48,6 & 15,7 & 15,7 & 11,1 \\
\hline Perales de Tajuña. & 1.821 & 39 & $-5,4$ & 26,3 & 32,3 & 15,7 \\
\hline Pezuela T. & 516 & 50 & $-43,4$ & 25,6 & 28,3 & 19,5 \\
\hline Pinilla del Valle & 134 & 90 & $-54,4$ & 33,3 & 0 & 25,1 \\
\hline Pinto $\ldots \ldots$ & 18.589 & 21 & 246,8 & 3,5 & 56,4 & 4,8 \\
\hline Piñuecar. & 151 & 85 & $-46,1$ & 43,7 & 0 & 32,9 \\
\hline Pozuelo de Alarcón & 31.228 & 12 & 231,8 & 3,3 & 24,4 & 6,5 \\
\hline Pozuelo R. . . . . . . . & 206 & 39 & -48 & 33,9 & 29,6 & 16,5 \\
\hline Prádena R. ....... & 106 & 90 & $-58,9$ & 50 & 0 & 25,5 \\
\hline Puebla S. & 58 & 98 & $-81,6$ & 50 & 0 & 33,7 \\
\hline Puentesviejas & 443 & 70 & $-39,7$ & 23,9 & 35,1 & 20,7 \\
\hline Quijorna & 505 & 39 & $-10,8$ & 17,7 & 11,8 & 12,9 \\
\hline Rascafría & 1.130 & 94 & $-19,6$ & 24,9 & 11,6 & 15,2 \\
\hline Redueña & 89 & 53 & $-23,3$ & 28,6 & 28,6 & 26 \\
\hline Rivatejada & 297 & 42 & 1,4 & 31,5 & 5,3 & 13,5 \\
\hline Rivas-Vaciamadrid .... & 652 & 19 & -46 & 51 & 14,6 & 9,4 \\
\hline Robledillo & 77 & 66 & $-56,7$ & 28,5 & 14,3 & 25,7 \\
\hline Robledo de Chavela. & 1.620 & 65 & 7,4 & 14,9 & 5,5 & 12,2 \\
\hline Robregordo $\ldots \ldots \ldots$ & 101 & 91 & $-65,9$ & 28,5 & 0 & 22,4 \\
\hline Rozas M. .... & 13.513 & 18 & 324,3 & 5,7 & 17,8 & 8,3 \\
\hline Rozas $\mathrm{P}$. & 420 & 82 & -17 & 68,3 & 0 & 18,3 \\
\hline San Agustín & 1.920 & 34 & 205,7 & 15 & 37 & 5,6 \\
\hline S. Fernando de Henares. & 19.310 & 17 & 378,8 & 2,5 & 52,9 & 3,7 \\
\hline S. Lorenzo del Escorial .. & 9.518 & 51 & 19,5 & 3,7 & 11,1 & 11,5 \\
\hline San Martin de la Vega. & 5.009 & 22 & 19,1 & 29,1 & 38,1 & 8,9 \\
\hline San Martin de Valdeig. & 4.786 & 67 & 19,8 & 25,8 & 15,8 & 13,9 \\
\hline San Sebt. de los Reyes & 39.866 & 18 & 1.090 & 2,8 & 34,1 & 3,7 \\
\hline Santa $\mathrm{M}^{\mathrm{a}}$ de la Alameda & 784 & 71 & $-21,1$ & 25 & 1,6 & 14,9 \\
\hline
\end{tabular}




\section{CUADRO III (Continuación)}

\begin{tabular}{|c|c|c|c|c|c|c|}
\hline MUNICIPIO & $\begin{array}{l}\text { POBLACION } \\
\text { TOTAL }\end{array}$ & $\begin{array}{l}\text { DISTANCIA } \\
\text { MADFID }\end{array}$ & $\begin{array}{c}\% \\
\text { INCREMENTO } \\
1960-81\end{array}$ & AGRICULTURA & $\begin{array}{c}\% \\
\text { INDUSTRIA }\end{array}$ & $\begin{array}{c}\% \\
\text { ANCIANOS }\end{array}$ \\
\hline Santorcaz & 657 & 40 & 11,2 & 22,7 & 27,3 & 14,6 \\
\hline Santos $\mathrm{H}$. & 875 & 45 & $-15,5$ & 16,1 & 21,4 & 14,9 \\
\hline Serna Mte. & 75 & 80 & -46 & 40,1 & 0 & 11,8 \\
\hline Serranillos & 384 & 30 & 12,6 & 31,8 & 22 & 11,8 \\
\hline Sevilla la Nueva ...... & 800 & 30 & 108,3 & 17,1 & 12,3 & 10,6 \\
\hline Somosierra. & 116 & 93 & $-39,3$ & 22,2 & 0 & 14,9 \\
\hline Soto del Real & 1.382 & 41 & 244,6 & 24,2 & 13,7 & 7,5 \\
\hline Talamanca del Jarama & 885 & 38 & 30,3 & 39,9 & 26,1 & 12,5 \\
\hline Tielmes ............. & 1.841 & 44 & 4,4 & 21,4 & 40,4 & 14,6 \\
\hline Titulcia & 835 & 35 & 1,6 & 61,3 & 13,6 & 18,3 \\
\hline Torrejón & 75.398 & 20 & 598,5 & 1,3 & 48,3 & 3,3 \\
\hline Torrejón de la Calzada & 738 & 26 & 190.5 & 22,4 & 46 & 5.8 \\
\hline Torrejón de Velasco & 1.275 & 28 & $-4,7$ & 48,9 & 20,7 & 13,5 \\
\hline Torrelaguna & 2.481 & 58 & $-1,1$ & 7,8 & 46,7 & 17 \\
\hline Torrelodones & 3.495 & 30 & 120,9 & 11,4 & 7,8 & 5,9 \\
\hline Torremocha & 173 & 63 & $-17,2$ & 43,5 & 45,2 & 21,4 \\
\hline Torre Alameda & 2.389 & 33 & 61,4 & 10 & 50,2 & 7,7 \\
\hline Valderacete .. & 766 & 57 & $-40,8$ & 20,6 & 14,8 & 20,2 \\
\hline Valdeavero. & 472 & 45 & $-8,3$ & 39,2 & 34,6 & 16.1 \\
\hline Valdelaguna. & 803 & 50 & $-15,1$ & 60,9 & 12,2 & 15,7 \\
\hline Valdemanco. & 446 & 62 & 13.5 & 11,1 & 3,7 & 17,9 \\
\hline Valdemaqueda & 390 & 71 & -15 & 24,2 & 0 & 9,1 \\
\hline Valdemorillo & 2.063 & 38 & 40,3 & 22,4 & 15,6 & 9 \\
\hline Valdemoro & 13.184 & 27 & 198.9 & 3,8 & 40,9 & 5,2 \\
\hline Valdeolmos & 448 & 36 & $-0,9$ & 40,7 & 18,5 & 16,8 \\
\hline Valdepiélagos & 317 & 45 & $-17,2$ & 54,7 & 17,4 & 17.3 \\
\hline Valdetorres del $\mathrm{J}$. & 1.127 & 39 & 14,6 & 30,9 & 15,6 & 12,2 \\
\hline Valdilecha...... & 1.438 & 38 & $-6,6$ & 35,1 & 18,2 & 16,9 \\
\hline Valverde $\ldots \ldots \ldots$ & 198 & 41 & $-40,4$ & 13,3 & 20 & 13,5 \\
\hline Velilla S. Antonio & 1.543 & 25 & 48,9 & 10 & 53,6 & 7,8 \\
\hline Vellón $(E \mathrm{El}) \ldots \ldots \ldots \ldots$ & 898 & 48 & $-9,3$ & 50,2 & 9,6 & 12,9 \\
\hline Venturada $\ldots \ldots \ldots \ldots$ & 295 & 51 & 84,4 & 50 & 25 & 12 \\
\hline Villa del Prado & 2.770 & 62 & 2,9 & 54,2 & 9,7 & 11,2 \\
\hline Villaconejos ... & 2.797 & 48 & $-10,7$ & 77,8 & 8,1 & 11,9 \\
\hline Villalbilla $\ldots \ldots \ldots \ldots$ & 994 & 38 & 14,4 & 27,1 & 27,1 & 8,9 \\
\hline Villamanrique de Tajo .. & 583 & 62 & $-37,5$ & 52,1 & 14,3 & 16,7 \\
\hline Villamanta $\ldots \ldots \ldots$ & 927 & 42 & $-8,4$ & 20,7 & 18,9 & 11,5 \\
\hline Villamantilla & 291 & 45 & $-29,7$ & 50 & 0 & 18,5 \\
\hline Villanueva de la Cañada & 1.997 & 36 & 237,3 & 20 & 12,6 & 6 \\
\hline Villanueva de Perales .. & 349 & 43 & $-12,3$ & 45,8 & 25,1 & 14 \\
\hline Villanueva del Pardillo .. & 993 & 26 & 103,5 & 8,7 & 28,2 & 7,5 \\
\hline Villar del Olmo & 471 & 48 & $-17,2$ & 35,7 & 9,9 & 16,2 \\
\hline Villarejo de Salvanés & 4.232 & 50 & -2 & 14,6 & 39,7 & 12,7 \\
\hline Villaviciosa de Odón. & 6.102 & 20 & 175,5 & 8,7 & 99,6 & 11,5 \\
\hline Villavieja de Lozoya & 161 & 58 & $-57,2$ & 20,2 & 20,2 & 22 \\
\hline Zarzalejo .......... & 819 & 58 & $-28,7$ & 17 & 49,5 & 16 \\
\hline
\end{tabular}


El primer paso consiste en hallar la correlación existente entre las seis variables (las cinco señaladas más el porcentaje de ancianos) con lo que obtenemos la siguiente matriz de correlación:

\section{CUADRO IV. MATRIZ DE CORRELACION}

\begin{tabular}{lcccccc}
\hline & POBLACION & DISTANCIA & INCR. POBL. & AGRICULT. & INDUSTRIA & ANCIANOS \\
\hline POBLACION & 1,00000 & $-0,20649$ & 0,07153 & $-0,13712$ & 0,06087 & $-0,06548$ \\
DISTANCIA & $-0,20649$ & 1,00000 & $-0,37606$ & 0,56471 & $-0,57783$ & 0,74444 \\
& & & & & & \\
INCR. POBL. & 0,07153 & $-0,37606$ & 1,00000 & $-0,32949$ & 0,25496 & 0,37551 \\
AGRICULT. & $-0,13712$ & 0,56471 & $-0,32949$ & 1,00000 & $-0,46979$ & 0,66800 \\
& & & & & & \\
INUUSTRI. & 0,06087 & $-0,57783$ & 0,25496 & $-0,46979$ & 1,00000 & $-0,41908$ \\
\hline ANCIANOS & $-0,06548$ & 0,74444 & $-0,37551$ & 0,66800 & $-0,41908$ & 1,00000 \\
\hline
\end{tabular}

Como puede verse en el cuadro IV la correlación existente entre el porcentaje de ancianos y la población es muy baja $(0,06)$. Con el incremento de población entre 1960 y 1981 sólo alcanza un valor de -0,37, también baja, aunque ya cercana al valor de 0,4 que se considera de correlación media. Este valor lo superan las otras tres, aunque con la población activa industrial tampoco es muy significativa $(-0,41)$. Con la distancia y con los activos agrarios la correlación es alta $(0,74$ y 0,66 respectivamente).

Para eliminar el efecto de la posible colinealidad entre algunas de las variables utilizadas, hemos realizado una matriz de correlación parcial, donde efectivamente se aprecia que la significación de correlación entre el porcentaje de ancianos y el de activos industriales es despreciable. 


\section{CUADRO V. MATRIZ DE CORRELACION PARCIAL}

\begin{tabular}{lcccccc}
\hline & POBLACION & DISTANCIA & INCR. POBL. & AGRICULT. & INDUSTRIA & ANCIANOS \\
\hline POBLACION & $-1,00000$ & $-0,24320$ & 0,00854 & $-0,11934$ & $-0,10483$ & 0,17683 \\
DISTANCIA & $-0,24320$ & $-1,00000$ & $-0,11135$ & $-0,02473$ & $-0,42522$ & 0,59170 \\
INCR. POBL. & 0,00854 & $-0,11135$ & $-1,00000$ & $-0,08271$ & 0,03183 & $-0,10020$ \\
AGRICULT. & $-0,11934$ & $-0,02473$ & $-0,08271$ & $-1,00000$ & $-0,25451$ & 0,46279 \\
INDUSTRIA & $-0,10483$ & $-0,42522$ & 0,03183 & $-0,25451$ & $-1,00000$ & 0,15102 \\
ANCIANOS & 0,17683 & 0,59170 & $-0,10020$ & 0,46279 & 0,15102 & $-1,00000$ \\
\hline
\end{tabular}

Por tanto, desde este momento despreciamos las variables de poca correlación y proseguimos con la distancia y el porcentaje de activos a grícolas aplicando el análisis de regresión múltiple con las tres variables.

Realizado un primer análisis de regresión lineal múltiple entre el porcentaje de ancianos tomado como variable dependiente y la distancia y el porcentaje de activos agrícolas como variables independientes, obtenemos los siguientes resultados:

\begin{tabular}{lcc}
\hline \multicolumn{1}{c}{ VARIABLE } & COEFICIENTE & ERROR STANDARD \\
\hline CONSTANTE $\ldots \ldots \ldots \ldots \ldots$ & 1,116758 & 0,82089 \\
DISTANCIA $\ldots \ldots \ldots \ldots \ldots$ & 0,188717 & 0,019124 \\
\% ACT AGRIC. $\ldots \ldots \ldots \ldots$ & 0,1389 & 0,020875 \\
\hline
\end{tabular}

Por tanto:

$$
y=1,117+0,189 x_{1}+0,139 x_{2}
$$


Donde:

$$
\begin{aligned}
& y=\% \text { de ancianos } \\
& x_{1}=\text { distancia } \\
& x_{2}=\% \text { de activos agrícolas }
\end{aligned}
$$

\begin{tabular}{lccrc}
\hline & R & $R^{2}$ & \multicolumn{1}{c}{$F$} & \% VARIANZA \\
\hline DISTANCIA $\ldots \ldots \ldots \ldots \ldots$ & 0,744 & 0,55 & 272,59 & 74,4 \\
\% DE ACT. AGRIC. ................ & 0,668 & 0,45 & 44,27 & 66,8 \\
\hline
\end{tabular}

Que demuestra una buena adecuación de los datos al modelo.

En el gráfico 2 hemos realizado la representación gráfica en el espacio $\mathrm{R}^{3}$ de la nube de puntos correspondiente a los datos de las tres variables.

Analizando con el método de regresión lineal simple de forma independiente cada una de las variables distancia y porcentaje de activos agrícolas con el porcentaje de ancianos, comprobamos que se ajustan en ambos casos a rectas (gráficos 3 y 4) cuyas ecuaciones son:

$$
y=1,395+0,26 x_{1} \quad y=6,96+0,25 x_{2}
$$

SIMPLE REGRESSION OF ANCIANS ON DISTANCE

\begin{tabular}{lrrrr} 
PARAMETER & ESTIMATE & $\begin{array}{c}\text { STANDARD } \\
\text { ERROR }\end{array}$ & $\begin{array}{c}\text { T } \\
\text { VALUE }\end{array}$ & $\begin{array}{c}\text { PROB. } \\
\text { LEVEL }\end{array}$ \\
\hline INTERCEPT $\ldots$ & 1,39474 & 0,91508 & 1,52417 & 0,129262 \\
SLOPE $\ldots \ldots$ & 0,260575 & 0,0176163 & 14,7917 & 0 \\
\hline
\end{tabular}


$M^{a}{ }^{a}$ JOSÉ AGUILERA ARILLA Y M. ${ }^{a}$ PILAR GONZÁLEZ YANCI

ANALYSIS OF VARIANCE

\begin{tabular}{lrrrr}
\hline SOURCE $\ldots .$. & Sum of Squares & Df & Mean Square & F-Ratio \\
MODEL $\ldots \ldots$ & 5600,0231 & 1 & 5600,0231 & 218,7944 \\
ERROR $\ldots \ldots$ & 4504,7045 & 176 & 25,5949 & \\
\hline TOTAL & 10104,728 & 177 & \\
(CORr.) & & & \\
CORRELATION COEFFICIENT $=0,744445$ & & \\
STND. ERROR OF EST. $=5,05914$ &
\end{tabular}

SIMPLE REGRESSION OF ANCIANS ON AGRICULT.

\begin{tabular}{lrccc} 
PARAMETER & ESTIMATE & $\begin{array}{c}\text { STANDARD } \\
\text { ERROR }\end{array}$ & $\begin{array}{c}\text { T } \\
\text { VALUE }\end{array}$ & $\begin{array}{c}\text { PROB. } \\
\text { LEVEL }\end{array}$ \\
\hline INTERCEPT $\ldots \ldots$ & 6,96049 & 0,707231 & 9,84189 & $4,24105 E-14$ \\
SLOPE $\ldots \ldots \ldots$ & 0,255231 & 0,0214321 & 11,9088 & $7,54952 E-15$
\end{tabular}

ANALYSIS OF VARIANCE

\begin{tabular}{|c|c|c|c|c|}
\hline SOURCE ..... & Sum of Squares & Df & Mean Square & F-Ratio \\
\hline MODEL $\ldots \ldots$ & 4508,9915 & 1 & 4508,9915 & 141,8191 \\
\hline ERROR $\ldots \ldots$ & 5595,7361 & 176 & 31,7940 & \\
\hline $\begin{array}{l}\text { TOTAL } \\
\text { (Corr.) }\end{array}$ & 10104,728 & 177 & & \\
\hline \multicolumn{5}{|c|}{ CORRELATION COEFFICIENT $=0,668001$} \\
\hline \multicolumn{5}{|c|}{ STND. ERROR OF EST $=5,63861$} \\
\hline
\end{tabular}


Por lo tanto, como conclusión podemos decir que existe una relación clara entre la distancia, los activos agrarios y los ancianos en la Comunidad de Madrid, relación de signo positivo, es decir a más distancia mayor número de ancianos y de activos agrarios. En el trabajo que citamos al comienzo sobre el envejecimiento de los barrios de Madrid, llegamos también a una clara correlación entre el porcentaje de envejecimiento y la distancia al centro de la ciudad, pero en este caso se ajustaba a una curva de regresión de signo negativo (a mayor distancia menor porcentaje de ancianos). Ahora bien, esta relación no supone una relación causa-efecto del fenómeno, la distancia por sí misma no explica, sino que relacionadas con ella hay otra serie de variables que serán las que realmente expliquen la distribución espacial del envejecimiento, una de ellas es sin duda la cantidad de activos dedicados a la agricultura, otras pueden ser: la natalidad y la mortalidad, el nivel de renta, el volumen de jóvenes que emigran, la rentabilidad de la agricultura, los transportes, la accesibilidad, etc. En definitiva, en el caso de esta provincia, joven en su conjunto, es la influencia de la ciudad de Madrid la que condiciona la distribución del envejecimiento de su entorno. 


\section{BIBLIOGRAFIA}

Aguilera Arilla, M. ${ }^{a}$ J., Borderías, M. ${ }^{a}$ P., G. Yanci, M. ${ }^{a} P$. (en prensa): «El envejecimiento demográfico de los barrios de Madrid", Homenaje al profesor Casas Torres. Zaragoza.

Anuario Estadistico de la Comunidad de Madrid (1984), (1985), Madrid. 414 pp y 423 pp.

Atlas Básico del Area Metropolitana de Madrid. (1979) COPLACO, MOPU.

Bernabé Maestre, J. M. (1985): “Tendencias recientes en la población mundial. ¿Uno o varios tipos de transición demográfica?» Estudios Geográficos, XLVI, n. ${ }^{\circ}$ 178-179, pp. 83-101.

García Ballesteros, A. (1980 a): "Modificaciones en la estructura demográfica española como consecuencia del proceso de urbanización", Revista Internacional de Sociologia, n. ${ }^{\circ} 34$, páginas 111-122.

-: (1980 b): «Emigración y envejecimiento en la población rural de ambas Castillas y León", Revista Internacional de Sociología, n. ${ }^{\circ} 34$, pp. 333-344.

Garcia Ballesteros, A. Brandis Garcia, D. Troitiño Vinuesa, M. A. (1978): "Diferencias espaciales en el envejecimiento de la población rural española", $V$ Coloquio de Geografía. Granada, pp. 523-537.

Paillat, P. (1976): “Le viellissement de la France rural. Intensité, évolution, diffusion et typologie", Population, n. ${ }^{\circ} 6$, pp. 11471180 . 
PaILlat, P. (1987): «Régions à problemes démographiques concernant la structure de la population visant en particulier le viellissement". Actes du Séminaire sur les régions à problèmes démographiques en Europe. Strasbourg, PP. 303-398.

Paillat, P. et Parant, A. (1985): «Viellissement de la population et activités locals." Paris PUF (Trab. et doc. Ined. n. ${ }^{\circ} 109,318$ pp.). Géographie du viellissement et des personnes âgés en Grande Bretagne et France (Actes du Colloque de Londres, juillet 1986) Esp. Popul. Soc. 1987,2.

Trigo Portela, J. (1984): «Implicaciones económicas del envejecimiento de la población", Cuadernos de Economía, n. ${ }^{\circ} 33$, páginas 129-145. 


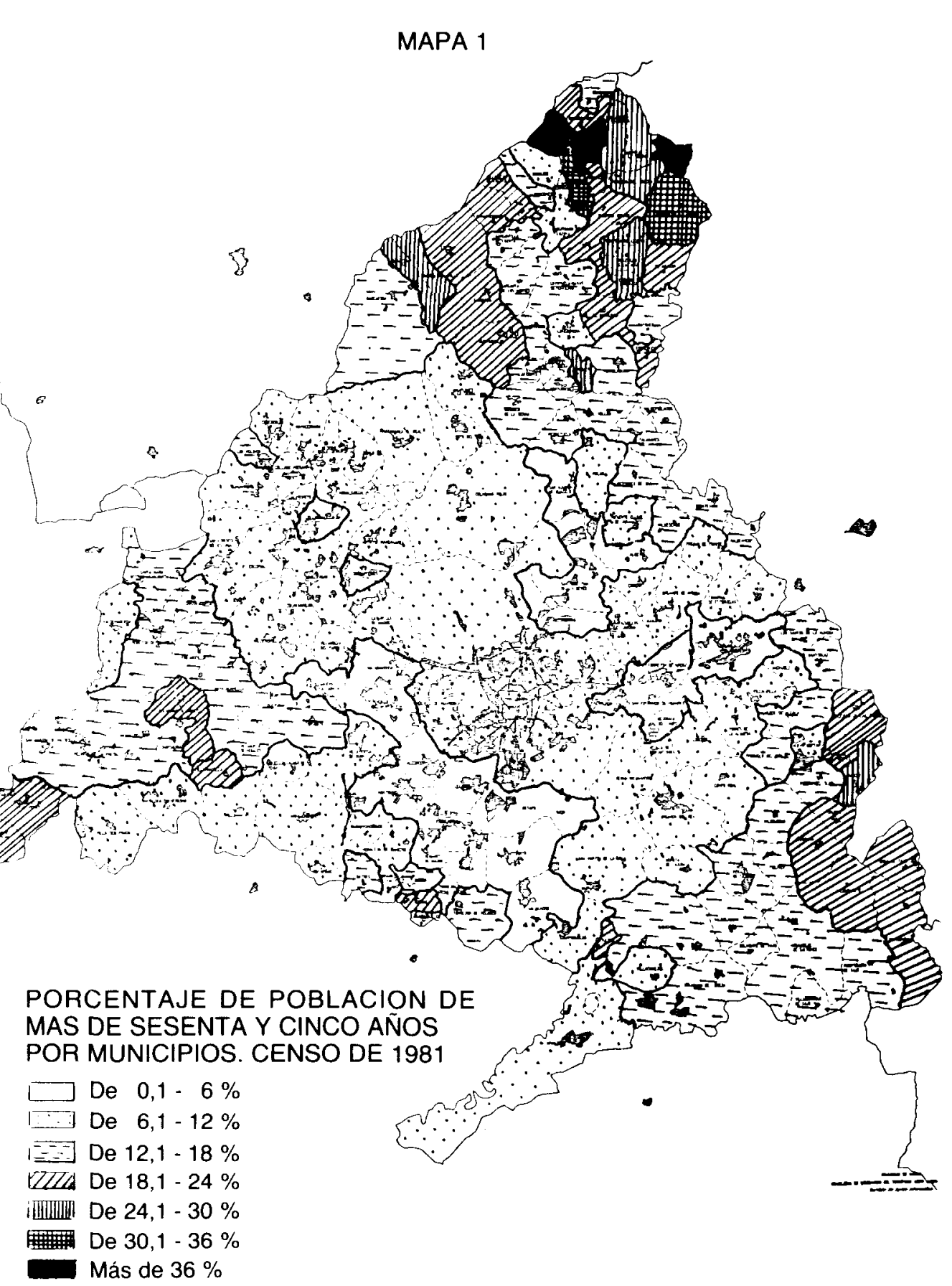


MAPA 2

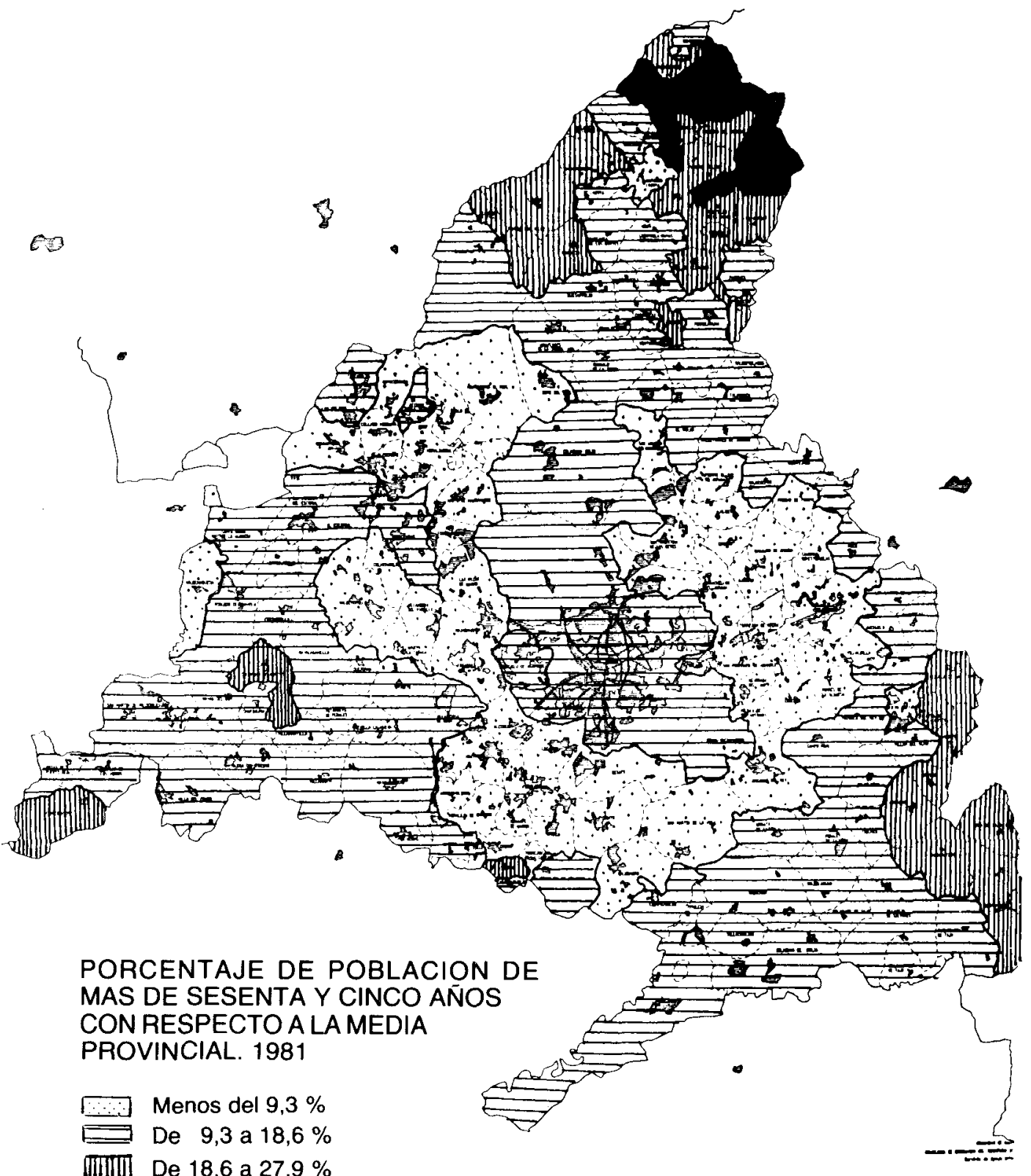




\section{MAPA 3}

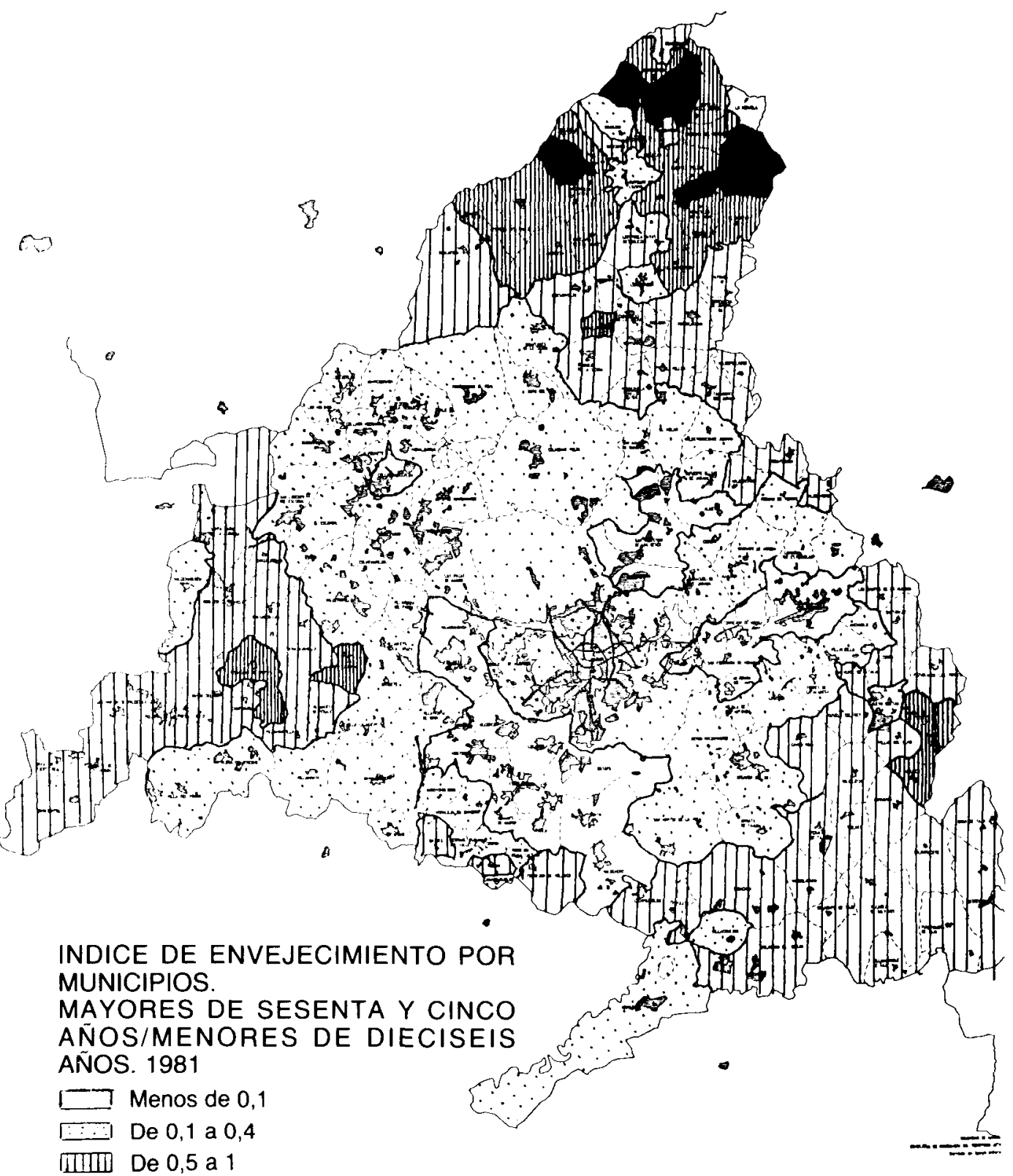

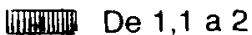

Más de 2 


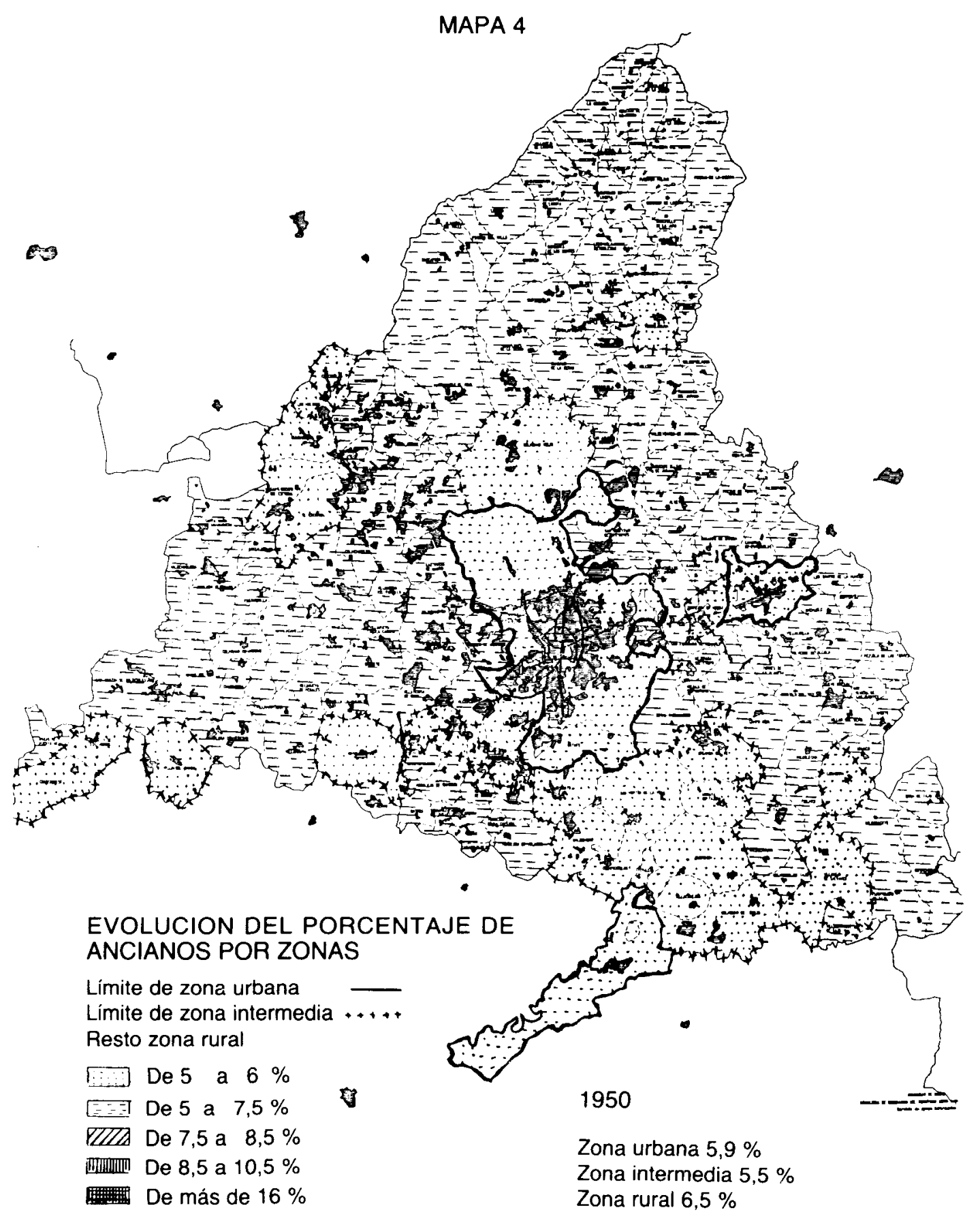




\section{MAPA 5}

6
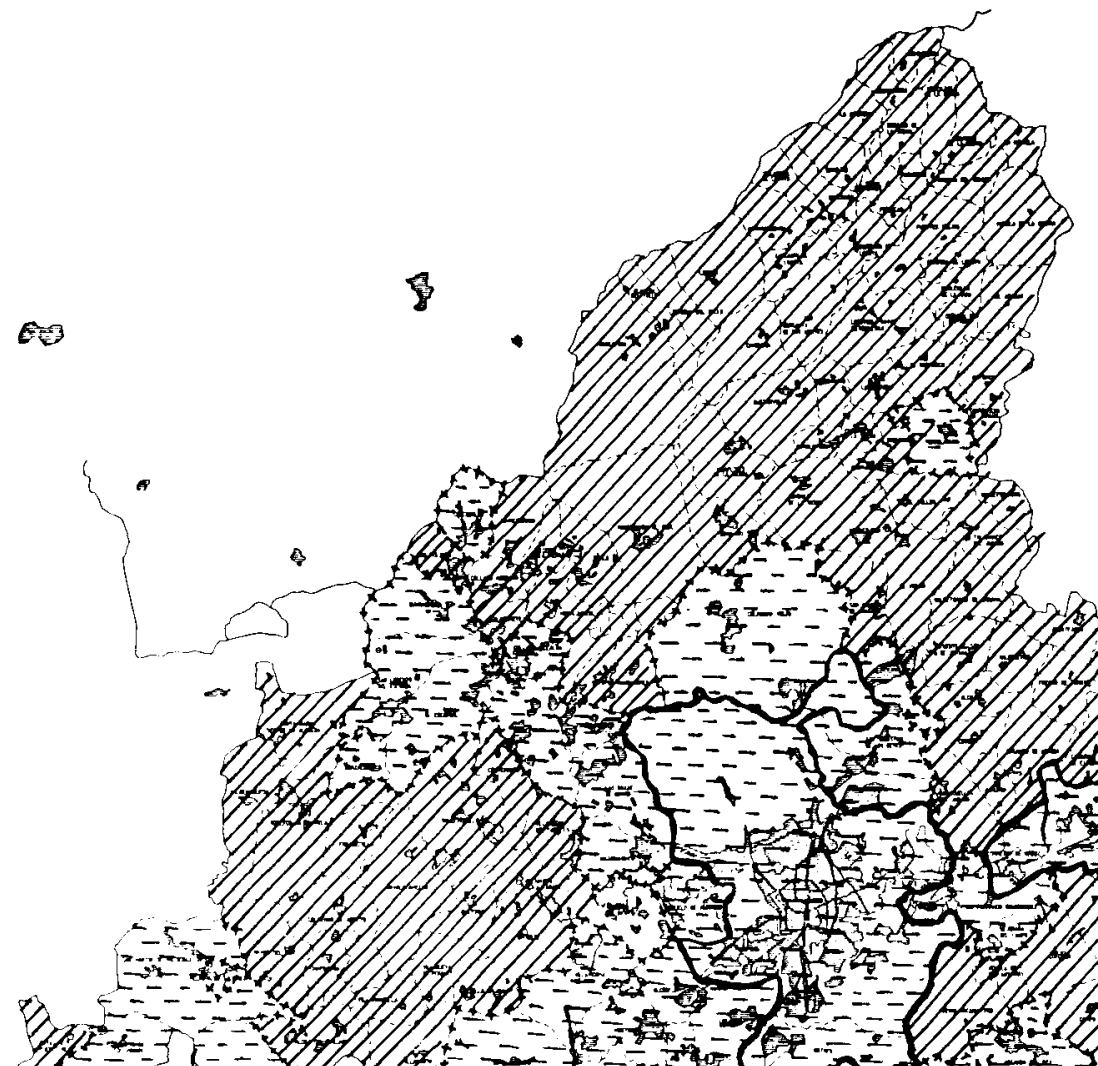

$x_{1}=-3(-2)$

$==0 \quad \mathrm{E}=0$
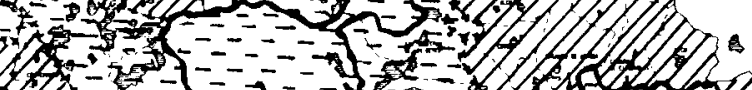

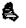
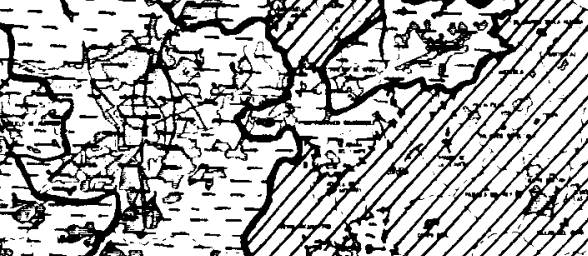

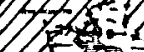




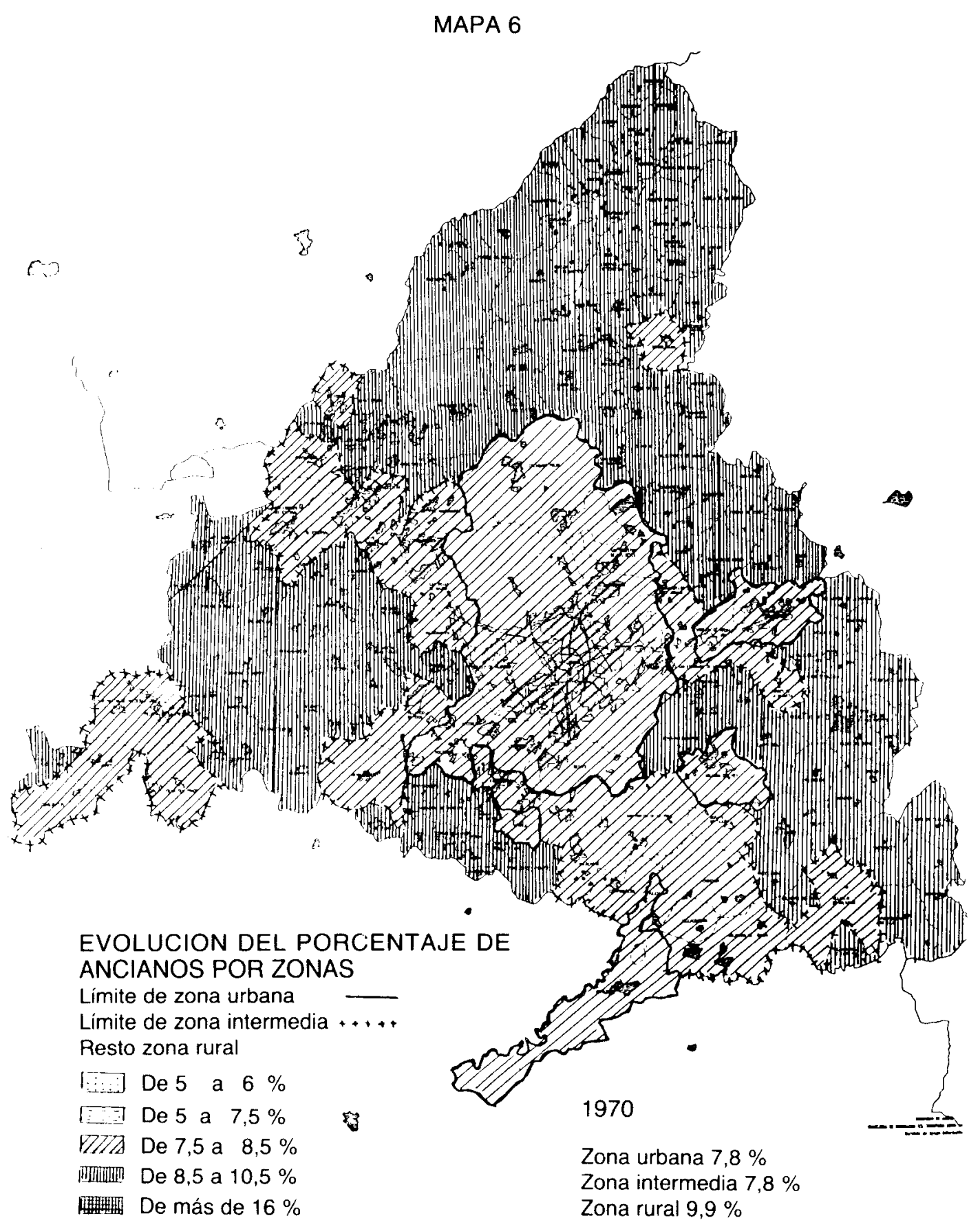




\section{La diferenciación espacial del envejecimiento demográfico}

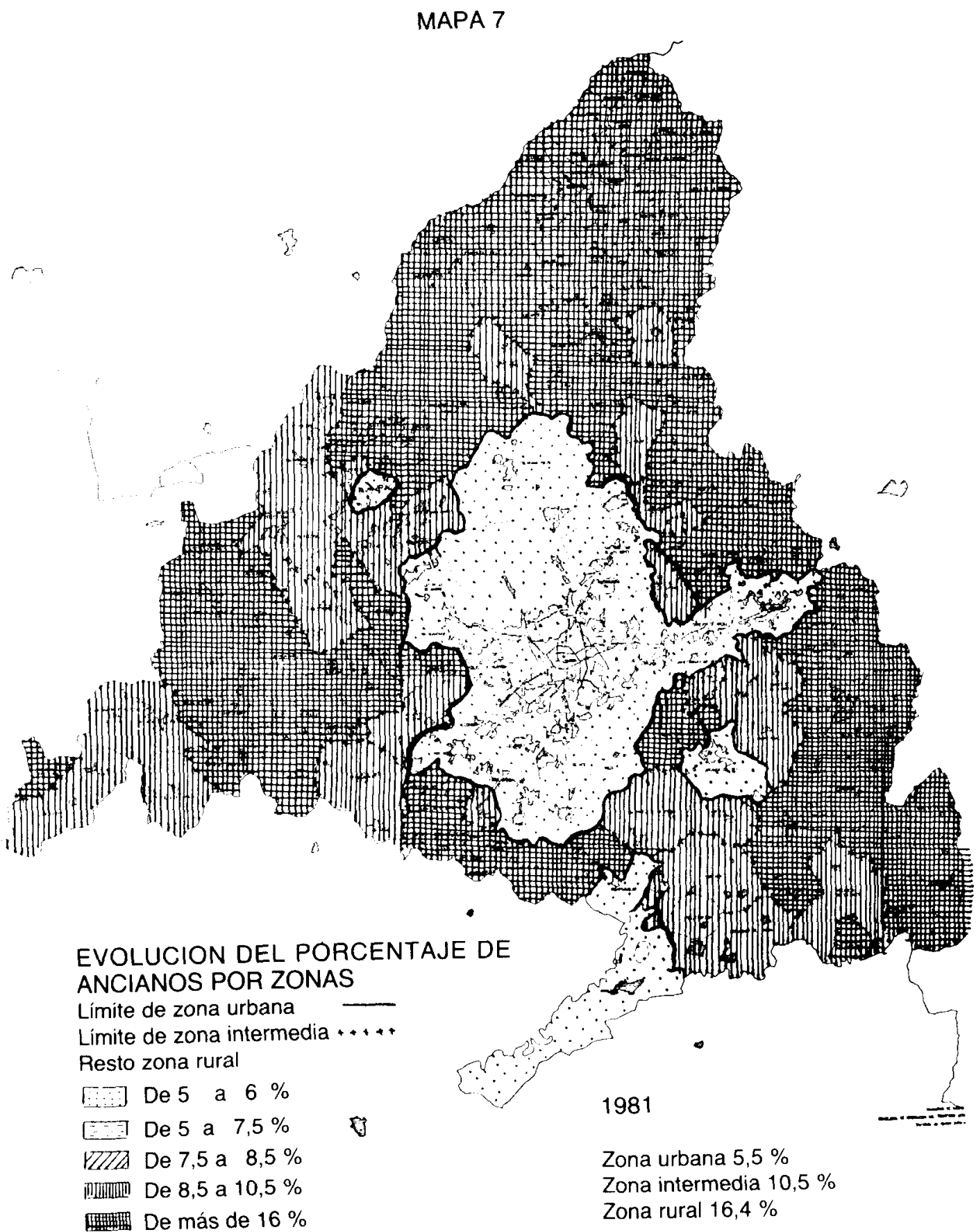




\section{MAPA 8}

$f$ 8
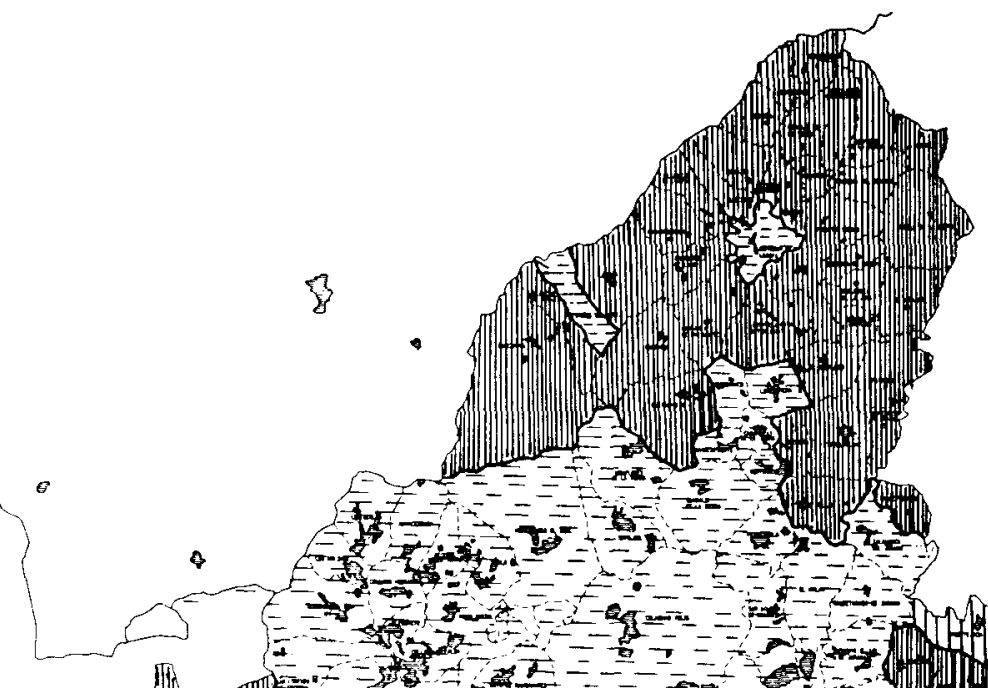

$\Rightarrow$ 㤢

(1) 


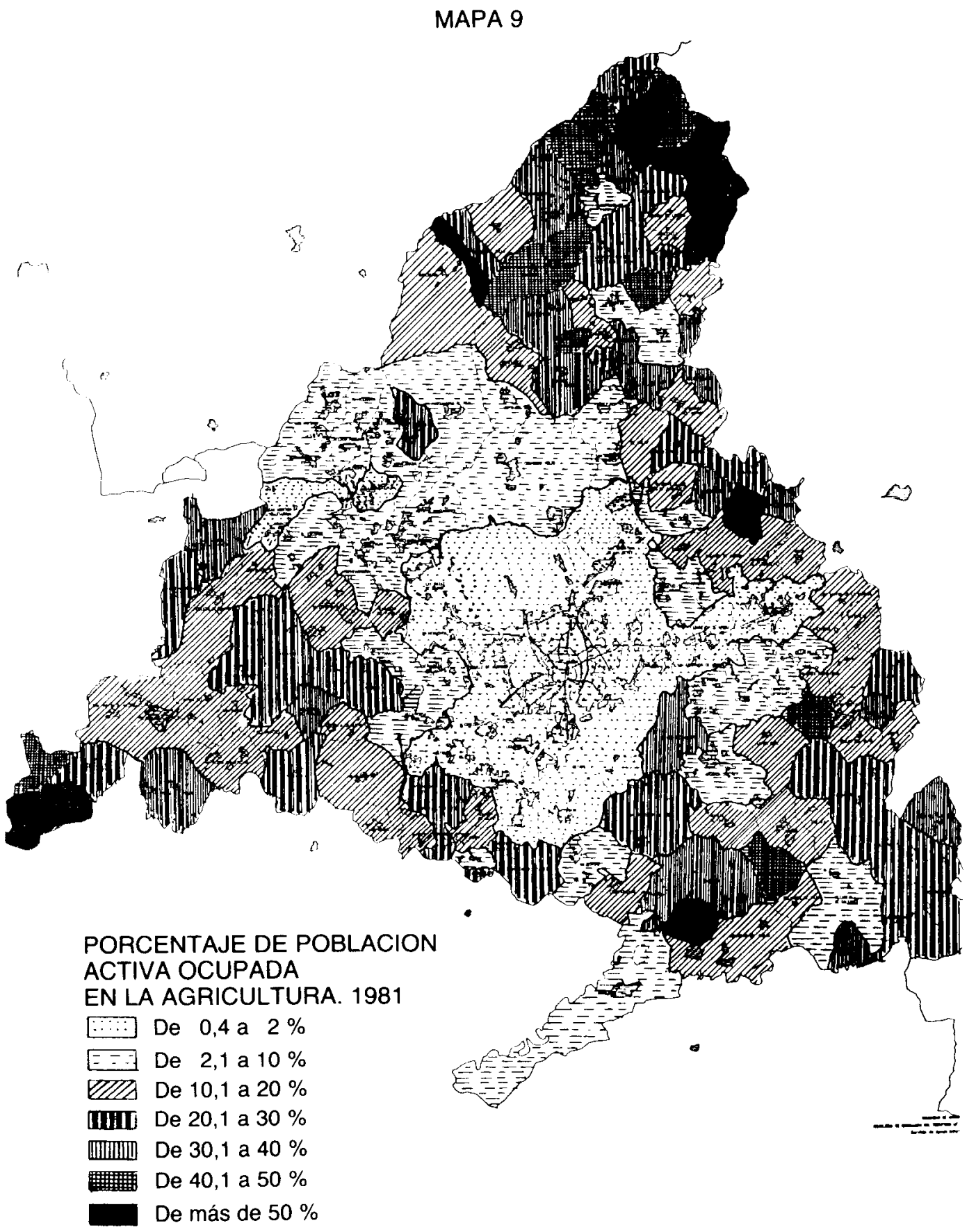


MAPA 10

$\varepsilon$
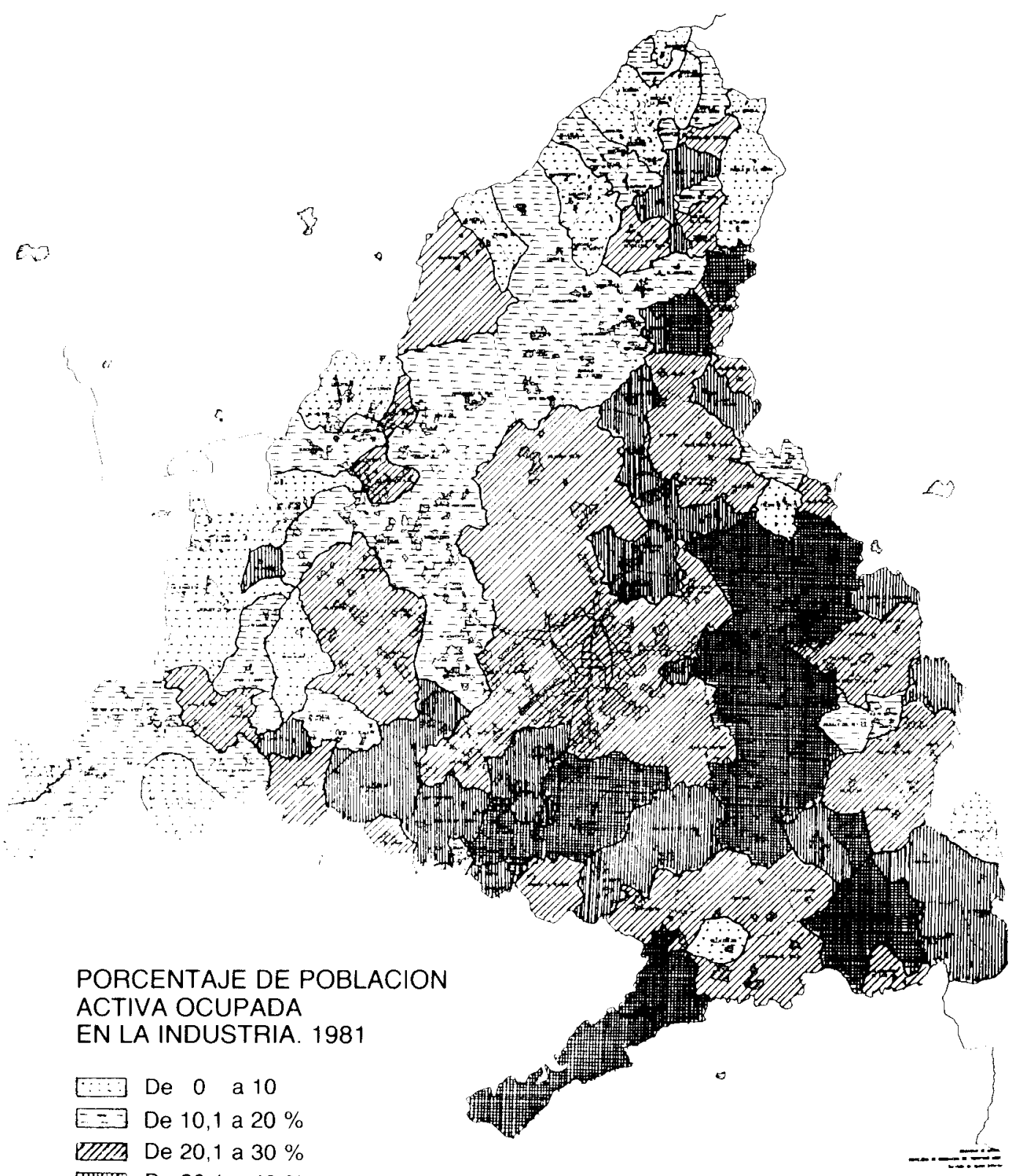

IIIIIIIII) De 30,1 a $40 \%$

De más de $40 \%$ 


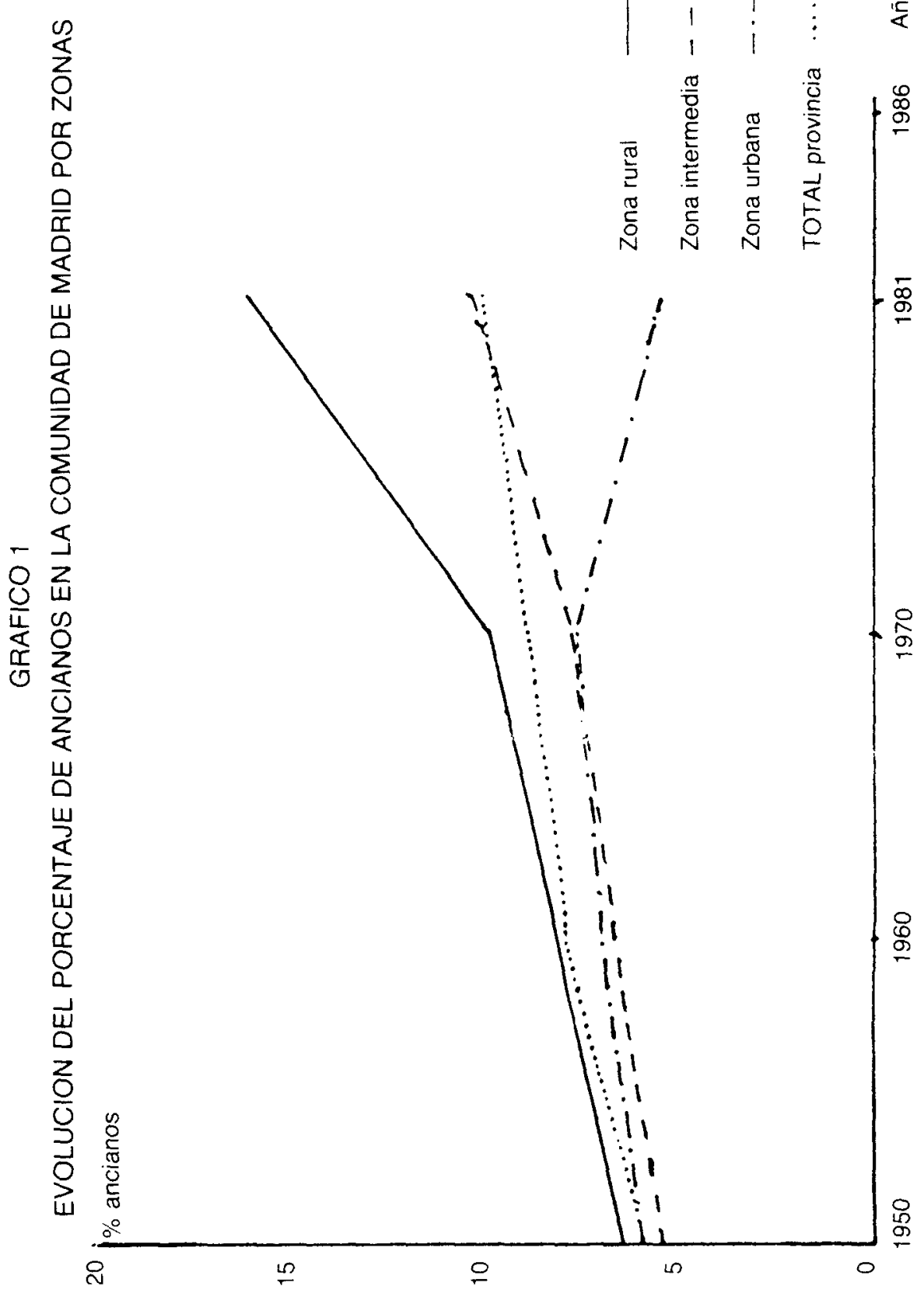


GRAFICO 2

REPRESENTACION GRAFICA EN EL ESPACIO R ${ }^{3}$ DE LA NUBE DE PUNTOS CORRESPONDIENTE A LOS DATOS DE LAS VARIABLES ANCIANOS, DISTANCIA Y AGRICULTURA

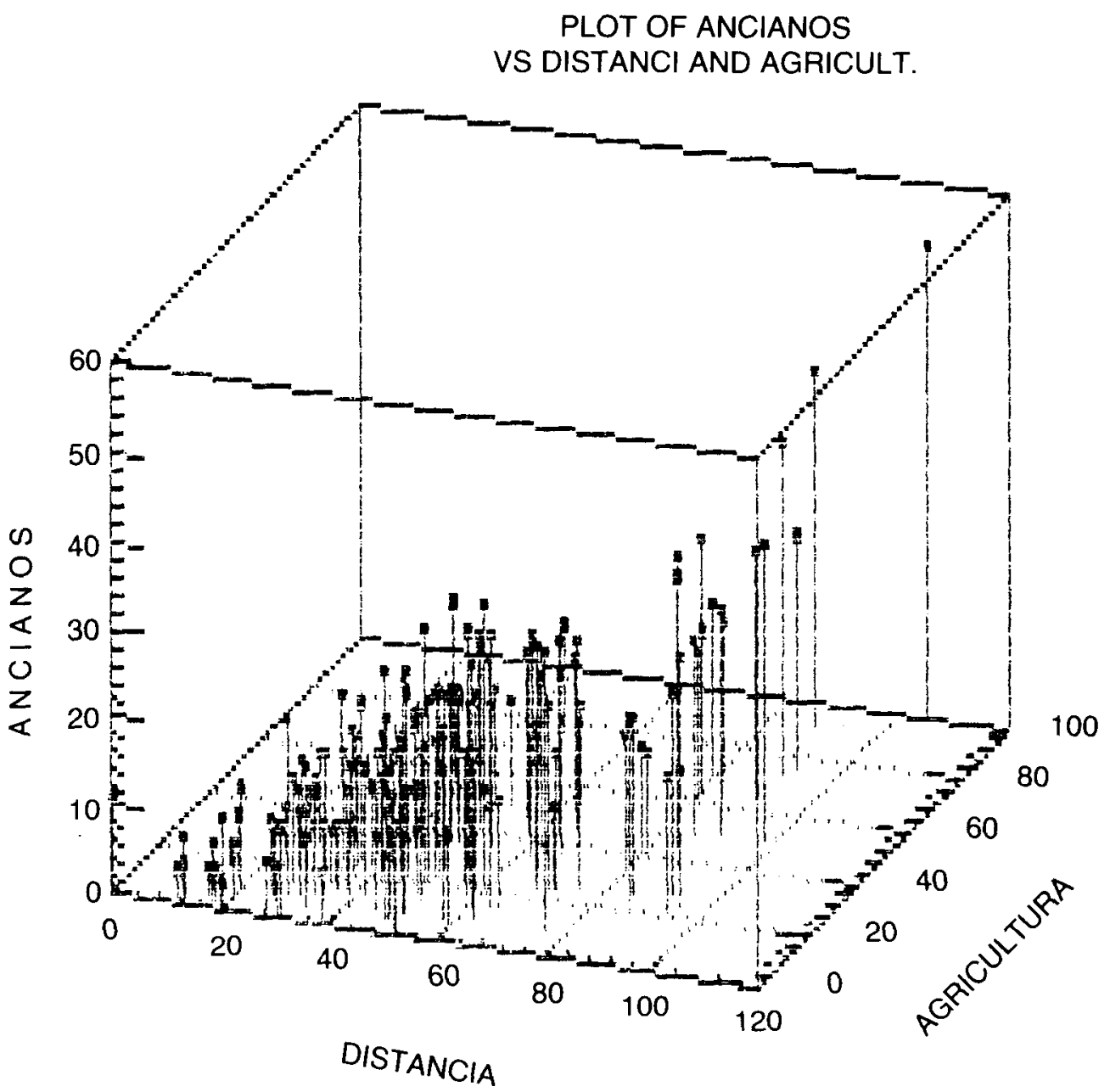


GRAFICO 3

REGRESSION OF ANCIANS ON DISTANCE

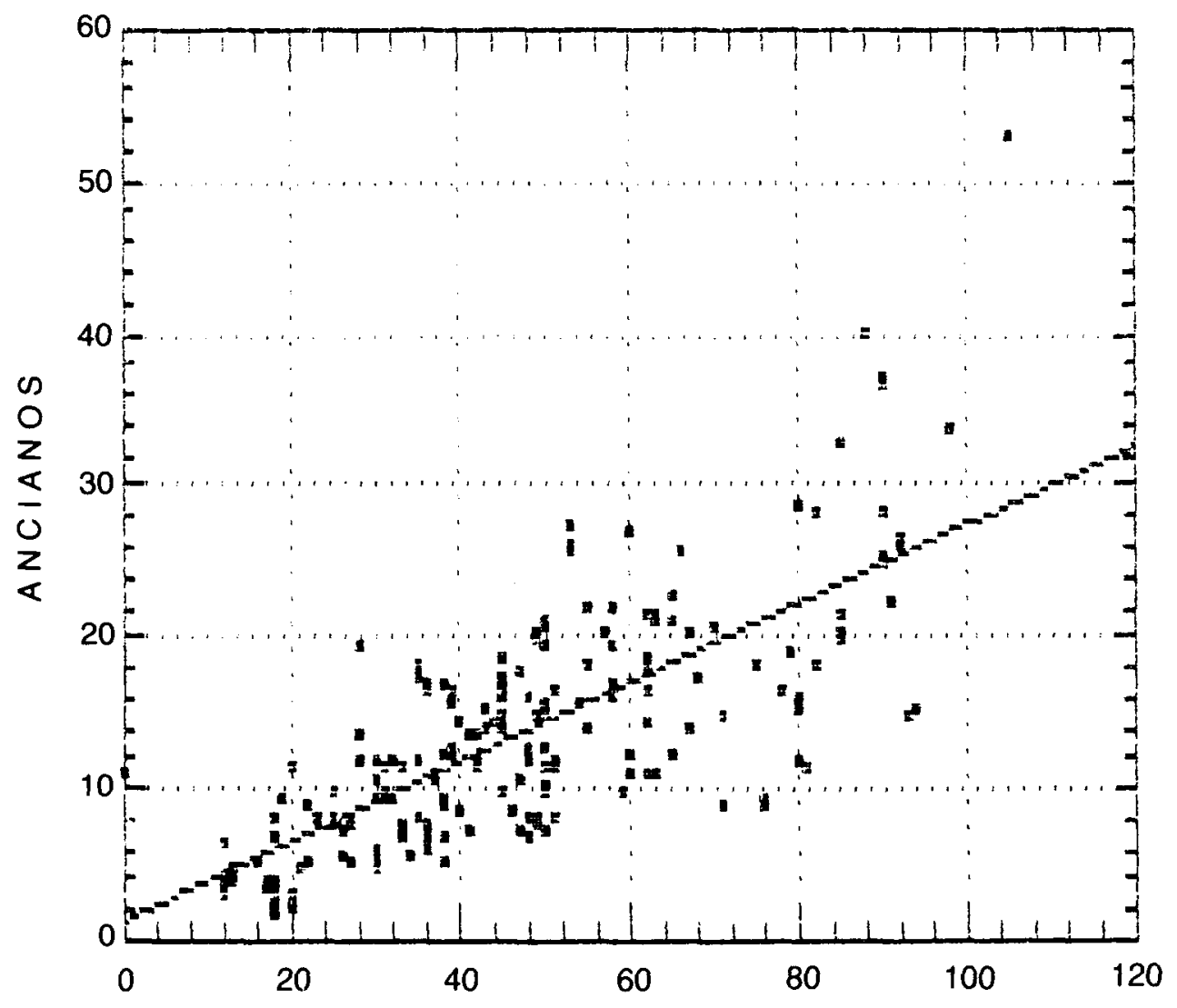

DISTANCIA 
GRAFICO 4

REGRESSION OF ANCIANS ON AGRICULT.

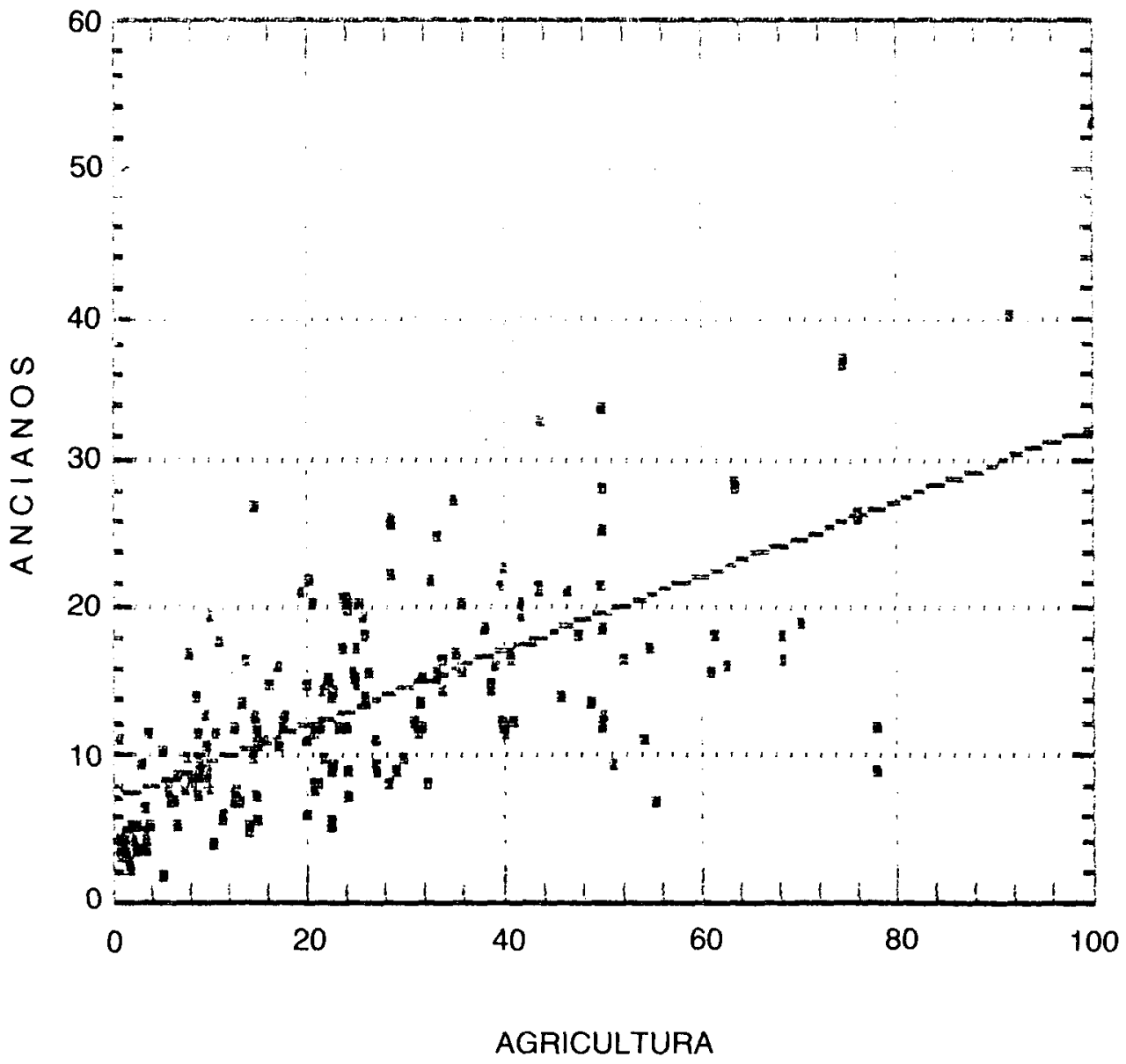

